\title{
Contribution of Work Ability and Core Self-Evaluations to Worker Health
}

\section{Karen Coomer and Jonathan Houdmont}

Accepted for publication in Occupational Medicine: $9^{\text {th }}$ May 2019

\begin{abstract}
Background: Work ability (WA) concerns the capacity to manage job demands in relation to physical and psychological resources. Core self-evaluations (CSE) refer to a composite personality construct comprising self-esteem, locus of control, self-efficacy, and emotional stability traits. Studies have shown the independent contribution of WA and CSE to work outcomes, yet none have explored their additive contribution, when applied together, to identify workers at risk of impaired health and performance-related outcomes.
\end{abstract}

Aims: To explore the contribution of WA and CSE to explaining variance in psychological distress and work engagement in a sample of UK manufacturing sector workers.

Method: A self-report questionnaire containing validated measures of WA, CSE, psychological distress, and work engagement was administered to employees in four UK manufacturing organisations. Bivariate correlations were calculated to identify patterns of relationships between the variables and hierarchical linear regression analyses performed to examine the incremental contribution of WA and CSE to the target variables.

Results: Analyses were conducted on data contributed by 311 workers ( $21 \%$ response rate). WA accounted for around one quarter of the variance in psychological distress and around 
one fifth of the variance in work engagement. The addition of CSE explained a further $10 \%$ (psychological distress) and 7\% (work engagement) of the variance.

Conclusions: These exploratory findings suggest that WA and CSE might be useful in the identification of workers at risk of poor psychological wellbeing and work effectiveness in UK manufacturing. Longitudinal sector-representative studies are required to establish the constructs' predictive power.

Key words: Work ability; core self-evaluations; psychological distress; work engagement; manufacturing. 


\section{Introduction}

Work ability (WA) refers to a worker's capacity to manage their job demands in relation to their health and mental resources [1]. It is a process that takes into account factors that may influence capacity and make the job more or less manageable [2]. This includes (i) health and functional capacity, (ii) education and competence, (iii) values and attitudes, and (iv) motivation that when considered in relation to work demands, work community and management, and the work environment results in an overall level of WA [2]. Many studies have explored relations between WA and worker health and performance-related constructs [3]. For instance, a study of middle-aged blue-collar male workers found associations between WA and health-related quality of life [4], while a large-scale study of Dutch employees found negative relations between WA and frequent and long-term sickness absence [5]. However, existing studies are inconsistent in terms of the treatment of WA as an independent versus dependent variable, and little research has been conducted in the context of the United Kingdom and, specifically, the manufacturing sector. This is an important omission given the ageing workforce and increasing requirement for longer working lives. There is limited knowledge about the potential utility of WA in this context for the identification of workers at risk for impaired health and performance related outcomes such as work engagement. In response to this knowledge gap, the first aim of the current study is to examine the contribution of WA to explaining variance in psychological distress and work engagement in the context of the UK manufacturing sector.

The core self-evaluations (CSE) composite personality construct was introduced in 1997 [6] to provide researchers and practitioners with a construct capable of predicting job satisfaction and other work-related outcomes [7]. The overarching CSE personality construct comprises four well-established traits: (i) self esteem, the overall value that one places on oneself as a person; (ii) generalized self-efficacy, an evaluation of how well one can perform 
across a variety of situations; (iii) neuroticism (emotional stability), the tendency to have a negative outlook; and (iv) locus of control, beliefs about the causes of event in one's life [7]. Taken together, these traits offer a fundamental appraisal of one's worthiness, effectiveness, and capabilities. Individuals with high self esteem, high self-efficacy, high emotional stability, and high locus of control are considered to have positive core self-evaluations and view themselves as capable, worthy and in control of their lives [8]. Since publication of the Core Self-Evaluations Scale (CSES) in 2003 [7] several studies have explored relations between the CSE personality construct and physical and psychological health and in doing so have highlighted the importance of self-concept to health functioning among workers [ 9,10$]$. In addition, a number of studies have explored the relationship between CSE and aspects of job performance [8]. For example, a significant association between CSE and employee engagement was demonstrated amongst hotel employees [11], while CSE was observed to moderate the relationship between work demands and depression among managers [12]. These studies offer support for the view that "employees with high core self-evaluations engage in more frequent goal setting, display greater effort and persistence toward achieving their goals, and capitalize on their opportunities and resources" [13]. In view of the growing body of evidence highlighting the role of the CSE personality construct in explaining worker health and performance-related outcomes, the second aim of this study is to examine whether CSE explained a significant amount of incremental variance in psychological distress and work engagement over and above that accounted for by WA.

\section{Methods}

A questionnaire was administered to manual workers in two large ( $>250$ employees) and two medium-sized (<249 employees) manufacturing organisations located in England and Scotland. They included a diverse array of manufacturing activities including food, 
electronics, and aeronautics. In each organisation human resources sponsored the study and arranged for the administration of questionnaires. Employees in two organisations had an occupational email address and an email was sent to these individuals containing an invitation to participate in the study and a link to an online questionnaire. Employees in the remaining organisations were issued with a paper-based invitation to participate and the questionnaire was appended to their monthly payslip. Responses were anonymous with paper questionnaires returned directly to the research team in a stamped addressed envelope. Ethical approval for the study was granted by a research ethics committee of the University of Nottingham.

The independent variables were WA and CSE. The first of these was measured using the Work Ability Index [14] that consists of seven dimensions: (i) current WA compared to lifetime best, (ii) WA as it relates to job demands, (iii) number of current diseases diagnosed by a physician, (iv) subjective estimation of work impairment due to disease, (v) sick leave over last year, (vi) own prognosis of WA two years from now, and (vii) mental resources. Scores are summed to create an overall score ranging from 7 to 49 , with a higher score indicating better WA. CSE was assessed using the 12-item Core Self-Evaluations Scale (CSES) [7] that measures the self-evaluation traits of self esteem, self-efficacy, emotional stability and locus of control. A sample item is "When I try, I generally succeed". Responses are given on a 5-point Likert-type scale with options ranging from strongly disagree (1) to strongly agree (5). Scores are summed, after reverse scoring of six negatively framed items, to create an overall score ranging from 12 to 60 , with a higher score indicating better CSE.

The dependent variables were psychological distress and work engagement. Psychological distress was measured using the 12-item General Health Questionnaire (GHQ12) [15]. A sample item is '[over the past few weeks have you] been able to concentrate on whatever you are doing?' Responses are given on a four-point scale of 'better than usual', 
'same as usual', 'less than usual', and 'much less than usual'. We used the GHQ scoring method (0-0-1-1) with points summed into a global score ranging from $0-12$ and higher scores indicating poorer psychological wellbeing. Work engagement was measured using the 9-item Utrecht Work Engagement Scale (UWES-9) [16] that consists of three sub-scales: vigour (e.g., 'at my work I feel bursting with energy'), dedication (e.g., 'my job inspires me'), and absorption (e.g., 'I am immersed in my work'). The items were rated on a 7-point scale of 0 (never) to 6 (daily), with scores summed to create an overall score out of a maximum possible score of 54 where higher scores indicated better work engagement. Basic demographic information was also collected (age and gender).

We performed analyses in SPSS V.20. Descriptive statistics and reliability coefficients were prepared for the study variables and bivariate correlations calculated to highlight the pattern of relationships between the independent and dependent variables. To examine the incremental contribution of WA and CSE to psychological distress and work engagement two hierarchical linear regression analyses were performed. The order in which the independent variables were entered into the regression analyses was based on the need to first identify the capacity of WA to account for explained variance in the target variables after controlling for the possible influence of background variables, and to explore additional variance explained by CSE over and above that accounted for by WA. Consequently, the first step contained age and gender, the second step consisted of WA, and the third step comprised CSE. A $p$ value of $<0.05$ was considered to be statistically significant throughout. G*Power [17] was used in order to determine the minimum sample size required for the study. For linear multiple regression a minimum sample size of 85 cases was required with alpha set at the $5 \%$ level $(\alpha=.05)$, power at $.8(\pi=0.80)$, a minimum effect size of moderate strength $\left(f^{2}=.15\right)[18]$, and four predictor variables. Pre-analysis checks on the data were undertaken 
and showed that there were no outliers, multicollinearity was not evident, and there were no major violations of the assumptions of normality and linearity.

\section{Results}

A total of 333 respondents submitted a questionnaire. After removal of responses involving missing values analyses were conducted on 311 cases (21\% response rate). In view of the low response rate the possibility of non-response bias was examined [19] by comparing the first 50 responses submitted to the last 50 , the latter having been submitted after the final reminder and therefore considered a proxy for non-responders as they would have been so were it not for the final reminder. Across the independent and dependent variables no significant differences were observed, suggesting that response bias was not present.

The mean age of respondents was $41.88(S D=10.73)$ and $58 \%$ were male. Scale descriptives, reliabilities, and bivariate correlations between study variables are presented in Table 1. Correlation analyses identified significant associations of moderate strength [18] in the expected direction between WA and the target variables. CSE was strongly associated with psychological distress and moderately associated with work engagement. Age and gender were not significantly associated with psychological distress, while age demonstrated a significant association of mild strength with work engagement. All scale reliabilities exceeded the commonly held minimum threshold for acceptable internal consistency of .80 [20], with the exception of the multifaceted WA construct.

\section{[Insert Table 1 about here]}

Although the bivariate correlations lend support for the relevance of the independent variables to the psychological distress and work engagement of workers in the sample, they do not clarify the extent to which WA and CSE additively account for variance in the target variables after controlling for the influence of background demographic factors. Hierarchical 
multiple regression was therefore undertaken to respond to these aims of the study. Table 2 indicates that the demographic variables (Model 1) explained 2\% of the variance in psychological distress, with the vast majority of the explained variance accounted for by WA (Model 2), which explained 24\% extra variance as compared to Model 1. When CSE was added (Model 3) it accounted for an additional 10\% of the variance. The model explained a total of $36 \%$ of the variance in psychological distress with WA and CSE making a statistically significant contribution. Similar findings were observed for work engagement as the criterion variable: Table 2 indicates that the demographic variables (Model 1) explained $5 \%$ of the variance in work engagement, with the vast majority of the explained variance accounted for by WA (Model 2), which explained 18\% extra variance as compared to Model 1. When CSE was added (Model 3), it accounted for an additional $7 \%$ of the variance. The model explained a total of $30 \%$ of the variance in work engagement with all variables making a statistically significant contribution.

\section{[Insert Table 2 about here]}

\section{Discussion}

This study examined the contribution of WA and CSE to explaining variance in psychological distress and work engagement in a sample of UK manufacturing sector workers. Firstly, there were significant relationships between WA and the target variables, with WA accounting for around one quarter of the variance in psychological distress and around one fifth of the variance in work engagement. Secondly, CSE made a significant incremental contribution to explaining variance in the target variables over and above that explained by WA. Specifically, CSE explained an additional $10 \%$ and $7 \%$ of the variance in psychological distress and work engagement respectively. These findings suggest that in the 
UK manufacturing context administration of measures of WA and CSE might be effective in the identification of workers at risk for psychological distress and low work engagement.

However, caution must be exercised when drawing such conclusions owing to the cross-sectional design of the study; longitudinal replication studies are required in order to establish the predictive power of WA and CSE in relation to the outcomes under investigation. The cross-sectional design used in the current study might also have raised the possibility of common method variance. If present, the correlations between the measures will be artificially inflated because participants will have applied the same biases when responding to each measure. However, the different patterns and direction of results observed across the study variables suggest that common method variance is an unlikely explanation for the results. The low response rate achieved in our study highlights the possibility of nonresponse bias. We assessed this by comparing the first 50 responders to the last 50 that responded after a final reminder had been issued and who would otherwise not have responded (thus forming a proxy non-response group), and found no significant differences. Nevertheless, the possibility cannot be discounted that non-responders differed significantly from responders on the variables under investigation. Future studies should seek to utilise sector-representative samples in order that findings might be generalised with confidence.

Our findings are in line with those of previous studies concerning the independent contribution of WA and CSE to worker health and performance-related outcomes [2-13], suggesting that these constructs may constitute valuable risk markers for psychological distress and work engagement that could provide the focus of targeted interventions. These findings suggest that interventions targeted at WA and potentially modifiable traits within the CSE construct might contribute to promoting psychological health and work engagement. However, the multifaceted nature of WA and CSE makes it difficult to gauge where interventions should be targeted to be optimally effective; further research is required to 
explore which elements of WA and traits that comprise CSE offer the most scope as intervention targets. Traits that make the largest contribution to explaining variance in healthand performance-related variables and are easily modifiable would be optimal intervention candidates. To date, interventions concerned with WA have treated the construct as an outcome variable rather than a direct intervention target [e.g. 21-22], while the notion of CSE as an intervention target remains conceptual.

Our findings have a number of theoretical and practical implications. Firstly, they may help to reinforce the importance of self-concept to work outcomes, and in doing so encourage researchers to take account of such factors in their studies so as to better understand the aetiology of worker health and performance. Secondly, the results may highlight the utility of the combined application of measures of WA and CSE for the identification of workers at risk for impaired psychological health and performance-related outcomes within the manufacturing context. In doing so, the findings might encourage uptake of these constructs in occupational health as an efficient and non-intrusive means by which to identify workers that might benefit from interventions to promote their health and organisational contribution. However, before definitive conclusions can be drawn on the utility of these measures further longitudinal and sector-representative research is required. 


\section{What is already known about this subject?}

- Work ability has been shown to contribute to explaining worker health and performance. Existing studies are inconsistent in terms of the treatment of work ability as an independent versus dependent variable, and little research has been conducted in the United Kingdom and, specifically, the manufacturing sector.

- Studies have likewise explored relations between the core self-evaluations personality construct and worker health and performance and in doing so highlighted the importance of self-concept to health functioning among workers.

- Evidence highlights the contribution of work ability and core self-evaluations to explaining worker outcomes. This study examined the additive contribution of work ability and core self-evaluations to explaining variance in psychological distress and work engagement.

\section{What this study adds}

- $\quad$ This study found a significant negative association of moderate strength between work ability and psychological distress and a significant positive correlation between work ability and work engagement.

- $\quad$ Core self-evaluations were strongly negatively associated with psychological distress and moderately positively associated with work engagement.

- Work ability explained around one quarter of the variance in psychological distress and one fifth of the variance in work engagement in UK manufacturing sector workers. Core self-evaluations contributed significant additional explanatory power to the statistical model.

\section{What impact this may have on practice or policy}


- These findings reinforce the importance of self-concept to work outcomes and in doing so may encourage researchers and practitioners to take account of such factors so as to better understand the aetiology of worker health and performance.

- The results highlight the utility of the combined application of measures of work ability and core self-evaluations for the identification of workers at risk for impaired psychological health and performance-related outcomes within the UK manufacturing sector.

- The findings may encourage uptake of these constructs in occupational health provision within UK manufacturing as an efficient and non-intrusive means by which to identify workers that might benefit from interventions to promote their health and organisational contribution. 


\section{References}

1. Coomer K, Houdmont J. Occupational health professionals' knowledge, understanding and use of work ability. Occup Med (Lond) 2013;63:405-409.

2. $\quad$ Ilmarinen J. Aging Workers. Occup Environ Med 2001;58:546-552

3. Van den Berg, T., Elders, L., de Zwart, B., \& Burdorf, A. The effects of work-related and individual factors on the Work Ability Index: a systematic review. Occup Environ Med, 2008;66:211-220.

4. Sorensen LE, Pekkonen MM, Mannikko KG, Louhevaara JS, Markku JA. Associations between work ability, health related quality of life, physical activity and fitness among middle-aged men. Appl Ergon, 2008;39:786-791.

5. Notembomer A, Groothoff J, van Rhenen W, Roelen C. Associations of work ability with frequent and long-term sickness absence. Occup Med (Lond) 2015;65:373-379.

6. Judge T, Locke E, Durham C. The dispositional causes of job satisfaction: A core evaluations approach. Res Organis Behav. 1997;19:151-188.

7. Judge T, Erez A, Bono, J, Thoresen C. The core self-evaluations scale: development of a measure. Personnel Psych 2003;56:303-331.

8. Judge T, Van Vianen A, De Pater I. Emotional stability, core self-evaluations, and job outcomes: A review of the evidence and an agenda for future research. Human Perf. $2004 ; 17: 325-346$.

9. Piccolo R, Judge T, Takahashi K, Watanabe N, Locke E. Core self-evaluations in Japan: relative effects on job satisfaction, life satisfaction, and happiness. J Organ Behav 2005;26:965-985.

10. Tsaousis I, Nikolaou I, Serdaris N, Judge T. Do the core self-evaluations moderate the relationship between subjective well-being and physical and psychological health? Personality Indiv Differences 2007;42:1441-1452.

11. Lee JJ, Ok CM. Drivers of work engagement: An examination of core self-evaluations and psychological climate among hotel employees. Int J Hosp Man 2015;44:84-98.

12. Hentrich S, Zimber A, Sosnowsky-Waschek N, Gregersen S, Petermann F. The role of core self-evaluations in explaining depression and work engagement among managers. Curr Psych 2017;36:516-529.

13. Grant AM, Wrzesniewski A. I wont let you down...or Will I? Core self evaluations, other-orientation, anticipated guilt and gratitude, and job performance. J of App Psych 2010; 95:108-121. 
14. Tuomi K, Ilmarinen J, Jahkola A, Katajarinne L, Tulkki A. Work Ability Index. 2nd revised ed. Helsinki. Finnish Institute of Occupational Health, 1998.

15. Goldberg D P and Williams PA. User's Guide to the GHQ. Windsor, NFER Nelson, 1988.

16. Schaufeli W, Bakker A, Salanova M. The Measurement of Work Engagement With a Short Questionnaire. Educ Psych Meas 2006;66:701-716.

17. Faul F, Erdfelder E, Lang A, Buchner A. G* Power 3: A flexible statistical power analysis program for the social, behavioral, and biomedical sciences. Behav Res Methods 2007;39:175-191.

18. Cohen J. Statistical power and analysis for the behavioral sciences (2nd ed.). Hillsdale, NJ: Lawrence Erlbaum, 1988.

19. Kerr R, McHugh M, McCrory M. HSE Management Standards and stress-related work outcomes. Occup Med (Lond) 2009;59:574-579.

20. Henson RK. Understanding internal consistency reliability estimates: A conceptual primer on coefficient alpha. Meas Evalu in Couns Dev 2001;34:177-189.

21. de Boer AG, Burdorf A, van Duivenbooden C, Frings-Dresen MH. The effect of individual counselling and education on work ability and disability pension: a prospective intervention study in the construction industry. Occup Env Med 2007;64:792-797.

22. Pohjonen T, Ranta R. Effects of worksite physical exercise intervention on physical fitness, perceived health status, and work ability among home care workers: Five-year follow-up. Prevent Med 2001;32:465-475. 


\section{Table 1}

Descriptive Statistics, Scale Reliabilities, and Correlations between Variables

\begin{tabular}{|c|c|c|c|c|c|c|c|c|}
\hline & $\mathrm{M}$ & SD & $\alpha$ & 1 & 2 & 3 & 4 & 5 \\
\hline 1. Age & 41.88 & 10.73 & -- & & & & & \\
\hline 2. Gender & -- & -- & -- & $.18 * *$ & & & & \\
\hline 3. Work ability & 42.84 & 5.13 & .72 & -.02 & .01 & & & \\
\hline 4. Core self-evaluations & 43.99 & 8.09 & .83 & $.13 *$ & .10 & $.49 * *$ & & \\
\hline 5. Psychological distress & 2.07 & 2.99 & .90 & -.11 & -.06 & $-.49 * *$ & $-.52 * *$ & \\
\hline 6. Work engagement & 35.57 & 10.19 & .93 & $.19 * *$ & -.04 & $.42 * *$ & $.45^{* *}$ & $-.32 * *$ \\
\hline
\end{tabular}

Note: Gender: $1=$ female, $2=$ male. $\alpha=$ Cronbach's alpha coefficient. ${ }^{*} \mathrm{p}<0.05,{ }^{*} \mathrm{p}<0.01$. 


\section{Table 2}

Hierarchical Regression Analyses Examining the Additive Contribution of Work Ability and Core Self-Evaluations on Psychological Distress and Work Engagement $(n=311)$

\begin{tabular}{|c|c|c|c|}
\hline & Model 1 & Model 2 & Model 3 \\
\hline Variable & \multicolumn{3}{|c|}{$\beta$} \\
\hline \multicolumn{4}{|c|}{ Psychological distress } \\
\hline Age & -.12 & $-.13^{*}$ & -.09 \\
\hline Gender & -.05 & -.04 & -.01 \\
\hline WA & & $-.50 * * *$ & $-.33 * * *$ \\
\hline CSE & & & $-.35 * * *$ \\
\hline$R^{2}$ & .02 & .26 & .36 \\
\hline$\Delta R^{2}$ & .02 & .24 & .10 \\
\hline F for change in $R^{2}$ & 2.62 & $93.54 * * *$ & $40.34 * * *$ \\
\hline \multicolumn{4}{|l|}{ Work engagement } \\
\hline Age & $.21 * * *$ & $.21 * * *$ & $.18^{* * *}$ \\
\hline Gender & -.09 & -.09 & $-.12 *$ \\
\hline WA & & $.43 * * *$ & $.29 * * *$ \\
\hline CSE & & & $.31 * * *$ \\
\hline$R^{2}$ & .05 & .23 & .30 \\
\hline$\Delta R^{2}$ & .05 & .18 & .07 \\
\hline F for change in $R^{2}$ & $6.93 * *$ & $69.81 * * *$ & $28.96^{* * *}$ \\
\hline
\end{tabular}

${ }^{*} \mathrm{p}<0.05, * * \mathrm{p}<0.01, * * * \mathrm{p}<0.001$.

$\beta$, standardised beta coefficient; $R^{2}$, explained variance; adj. $R^{2}$, adjusted explained variance. 